\title{
Numerical Simulation of Turbulent Flow Over Staggered Tubes Using Multi-Relaxation Time Lattice Boltzmann Method
}

\author{
JongWoon Park \\ Department of Nuclear and Energy Systems \\ Dongguk University \\ Republic of Korea
}

\begin{abstract}
A multi-relaxation time lattice Boltzmann method (MRT-LBM) fluid flow analysis code is developed based on a two-dimensional D2Q9 lattice model and sub-grid eddy viscosity model of Smagorinsky. Benchmarking of the MRT-LBM is performed for a turbulent flow over staggered tube bundles at Reynolds number of 18,000and compared with standard k- $\omega$ turbulence and large eddy simulation (LES). The accuracy of the present MRT-LBM with respect to the mean velocity profiles is found to be reasonably acceptable and even comparable to the LES whereas the computational efficiency is superior.
\end{abstract}

Keywords-staggered tube bundle; very high temperature reactor; multi-relaxation lattice Boltzmann method; large eddy simulation

\section{INTRODUCTION}

Turbulent flow over tube bundles has been of interest over several decades. Especially, inside a lower plenum of a very high temperature (nuclear) reactor (VHTR), hot gas jets from reactor fuel channels with very high velocities between 35 70 $\mathrm{m} / \mathrm{s}$ with temperatures of about $1,000 \mathrm{~K}$ mix with each other before flowing out to the exit and the analysis is a challenging task due to complex fluid flow physics such as unsteadiness, turbulence, buoyancy effects, strong streamline curvature, and formation of vortices, etc [1]. Therefore, efficiently running computational fluid dynamics (CFD) technique with reasonable accuracy would be desirable for a mechanical design.

Compared with previous studies [2-3] using general CFD methods or direct numerical simulation (DNS), Lattice Boltzmann Method (LBM) [4],as an alternative,is a relatively newer approach using simple microscopic models to simulate complicated macroscopic behavior of transport phenomena.

In this paper, a two-dimensional computational fluid dynamic analysis code is developed based on the multirelaxation time lattice Boltzmann method (MRT-LBM) derived from the generalized lattice Boltzmann equations (GLBE) of D'Humiereset al [5]. The code is a twodimensional D2Q9 lattice model and equipped with classical sub-grid eddy viscosity turbulence model of Smagorinsky [6]. The computational accuracy and efficiency of the code is benchmarked against a high Reynolds number flow with $\mathrm{Re}=18,000$ over multiple tube bundles of Simonin and Barcouda[7]. General purpose CFD computations are also performed using the FLUENT [8] for the same geometry and compared with the present MRT-LBM in terms of accuracy and computing time.

\section{Multi-RelaXATION TIME LATtiCE BOLtZMANN METHOD}

The multi-relaxation time lattice boltzmann method (MRTLBM) is developed by D'humières[5]. Two dimensional lattice version (D2Q9) is shown in fig. 1.

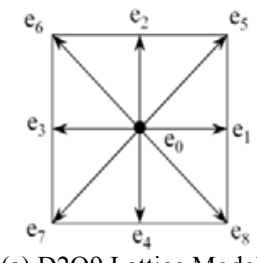

(a) D2Q9 Lattice Model

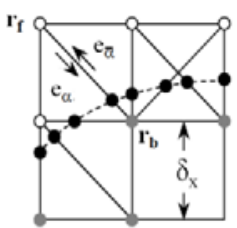

(b) Curved Wall Boundary

FIGURE I. MOMENTUM EXCHANGE MODEL FOR A CURVED WALL.

A set of velocity moments for this lattice model are defined as follows:

$$
\boldsymbol{m}=\left(\rho, e, \varepsilon, j_{x}, q_{x}, j_{y}, q_{y}, p_{x x}, p_{x y}\right)^{T}
$$

where $\rho$ is the fluid density, $e$ is the energy, $\varepsilon$ is a quantity related to the lattice velocity $e, j_{x}$ and $j_{y}$ are the components of the momentum flux density of $j=\rho \mathrm{u}\left(u_{x}, u_{y}\right), q_{x}$ and $q_{y}$ are the energy fluxes, $p_{x x}$ and $p_{y y}$ are the quantities proportional to the diagonal and off-diagonal components of the viscous stress tensor, and $T$ denotes the transpose operator. The set of velocity moments is related with the density distribution function $\boldsymbol{f}$ as following:

$$
\begin{gathered}
m=M f \\
f=\left(f_{0}, f_{1}, f_{2}, f_{3}, f_{4}, f_{5}, f_{6}, f_{7}, f_{8}\right)^{T}
\end{gathered}
$$

where $f_{\alpha}$ is the density distribution function moving with each discrete lattice velocities [9]ataspatial position and at time.

The matrix Min eqn(2) is the 9x9 matrix which linearly transforms the distribution function, $\boldsymbol{f}$, to the velocity momentset, $\boldsymbol{m}$, and is given by 


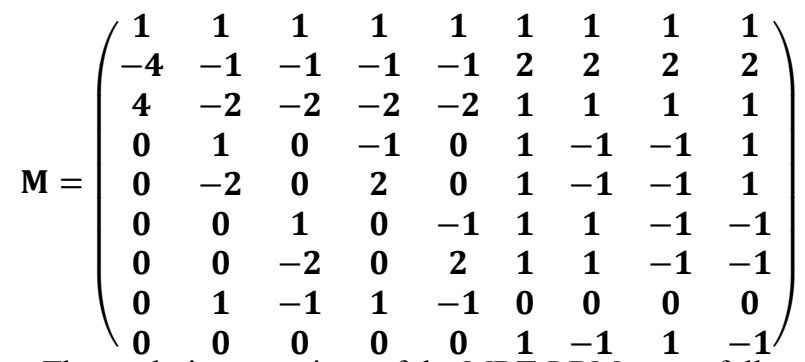

The evolution equations of the MRT-LBM are as follows:

$$
\boldsymbol{m}\left(r+e_{\alpha} \delta_{t}, t+\delta_{t}\right)-m(r, t)=-S\left[m(r, t)-m^{e q}(r, t)\right]
$$$$
\tilde{\boldsymbol{f}}_{\alpha}\left(\boldsymbol{r}+\boldsymbol{e}_{\alpha} \boldsymbol{\delta}_{t}, \boldsymbol{t}+\boldsymbol{\delta}_{t}\right)=-\mathbf{M}^{-1} \boldsymbol{m}\left(\boldsymbol{r}+\boldsymbol{e}_{\alpha} \boldsymbol{\delta}_{t}, t+\delta_{t}\right)
$$

whereSis the diagonal matrix consisting of nine relaxation parameters and $\boldsymbol{m}^{\boldsymbol{e q}}$ is the velocity moment equilibrium vector given by [5]

$$
m^{e q}=\left(0, e^{e q}, \varepsilon^{e q}, 0, q_{x}^{e q}, 0, q_{y}^{e q}, p_{x x}{ }^{e q}, p_{x y}{ }^{e q}\right)^{T}
$$

The macroscopic fluid density, $\rho$, and the moment fluxdensity, $J\left(j_{x}, j_{y}\right)$, can be obtained from a collision step at each time using the evolution equations, Eqs.(5) and (6), and given by:

$$
\begin{gathered}
\rho=\sum_{\alpha} f_{\alpha} \\
\boldsymbol{J}\left(j_{x}, j_{y}\right)=\sum_{\alpha} f_{\alpha} \boldsymbol{e}_{\boldsymbol{\alpha}}
\end{gathered}
$$

And new density distribution functions at the next time step are calculated from following streaming step:

$$
f_{\alpha}\left(r+e_{\alpha} \delta_{t}, t+\delta_{t}\right)=\tilde{f}_{\alpha}\left(r, t+\delta_{t}\right)
$$

where $\tilde{f}_{\alpha}$ denotes the post-collision distribution function calculated from eqn(6).

The unresolved turbulence scale is modeled by the standard Smagorinskymodel[6].The basic ideaof implementing turbulence in the LBM is to locally (i.e. independently for each site) increase relaxationtime residing in $S$ of eqn (4).

For the boundary conditions, the methods of Hoet al[10]andMei et al [11] are applied and all the equations are coded into the MATLABscripts[12]. Following is the discussion of the benchmarking analysis and it is made in terms of macroscopic flow quantities such as flow patterns and velocity profiles.

\section{BENCHMARKING ANALYSIS FOR STAGGERED TUBE BUNDLES}

Staggered tube bundles experimented by Simonin and Barcouda[7] is chosen as a benchmarking case. Arepeatingsubsection for computational simulationsconsists of a central tube and four quarter tubes in the corners as shown in fig. 2(a). The fluid flow is isothermal with Reynolds numberof around 18,000 .

To evaluate the performance of the present MRT-LBM over said general CFD methods, we also performed additional two computations: unsteady Reynolds-averaged Navier-Stokes (URANS) and large eddy simulation (LES). In case of the URANS, computations are performed using the second-order turbulence model, namely, the standard k- $\omega$ turbulence model. Secondly, the LES computations are madewithbothsub-grid scale (SGS) turbulence closures of Smagrinsky-Lilly [13] and WALE model [14].

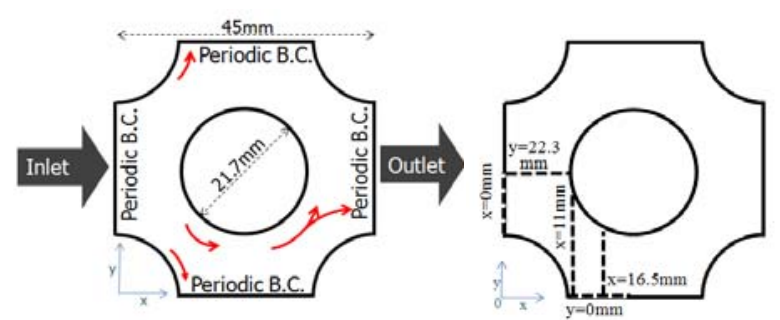

$\begin{array}{ll}\text { (a) Boundary conditions } & \text { (b) Lines where velcocitiesmeasured }\end{array}$ FIGURE II. SIMULATION CONDITIONS FOR THE FLOW THROUGH STAGGERED TUBE BUNDLES.

For the MRT-LBM computations, 140x140 lattices are used after grid sensitivity calculations. On the other hand, for the 2D Standard $\mathrm{k}-\omega$ model,42,952 cells were usedafter mesh sensitivity studies as well. In the 3D LES model, 1,788,584 cells in total were usedwhere the number of $2 \mathrm{D}$ cells is 47,068 and the number of $z$-direction cellsis 38 (domain length is 5 $\mathrm{mm}$ ). The initial inlet (upstream) velocity of $u_{m}=1.06 \mathrm{~m} / \mathrm{sec}$ is applied with water density of $998.2 \mathrm{~kg} / \mathrm{m}^{3}$ and dynamic viscosity of $0.001219 \mathrm{~kg} / \mathrm{m} . \mathrm{sec}$. For the other CFD calculations, the second-order implicit time differencing scheme is employed. The time step is set to be adaptive with the minimum time step set to $1 \times 10^{-5} \mathrm{sec}$ and the maximum set to $5 \times 10^{-3} \mathrm{sec}$. Total 30,000 time steps are computed.All the computations are carried out in the DELL T $4600^{\circledR}$ workstation with Intel ${ }^{\circledR} 6$-core $3 \mathrm{~Hz}$.

The unsteady flow velocity contours obtained from the four computations are shown in fig. 3. Among the four computations, only the flow structures from the URANS showstylized behaviour as shown in fig.3(b). However, for the rest of the computations (the MRT-LBM and the LES with two sub-grid models), shown in figs. 3(a), (c) and (d), respectively, one can clearly see more dynamically unstableturbulentstructures produced by the wake along the tubes. The flow structures from the LES-WALE shown in fig.3(d) are most chaotic and tattered than the other computations. And the flow structures from the MRT-LBM shown in fig.3(a) are most similar to those from the LESSmagorinsky shown in fig.3(c). Also, strong coupling of accelerated flow along the lower side of the central cylinder and decelerated flow along the upper rear side of the lateral cylinder (bottom left) is more evident in the MRT-LBM and the LEScomputations.Major reason of the difference between the URANS and the LES comes from averaging methods, e.g., time and spatial averaging, respectively.

The stream-wise time mean velocity (x-velocity, hereinafter) and span-wise time mean velocity (y-velocity, hereinafter)along the lines at $\mathrm{x}=0 \mathrm{~mm}, 11 \mathrm{~mm}, 16.5 \mathrm{~mm}, \mathrm{y}=0$ $\mathrm{mm}$ and $\mathrm{y}=22.5 \mathrm{~mm}$ shown in fig.2(b) are shown in fig. 4 . Overall results show that the discrepancies of the experimental and computational time-meanvelocities are marginal and probably in therange of the measurement uncertainty of the experiment. The MRT-LBM shows relatively greater deviation from the experiment near cylinder walls than the other general computations. This is due to the fact that the near-wall resolution is significantly higher in the URANS and the LES than the presentMRT-LBM which uses a simple bounce-back boundary model for the fluid-solid boundary. Nevertheless, $x-$ 
velocity profiles from the MRT-LBM are very close to the experimental data and comparable or even superior to the other computations.

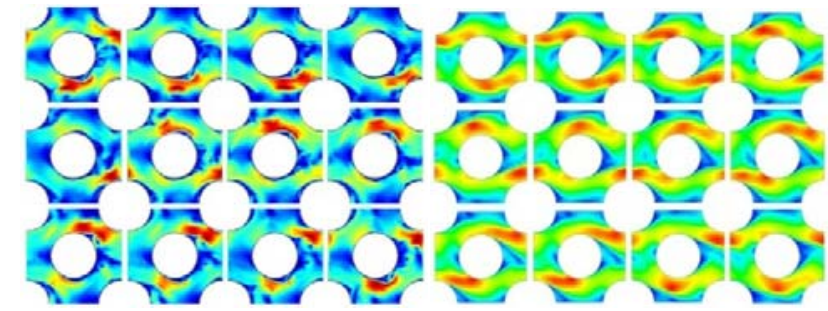
(a) MRT-LBM(b) URANS

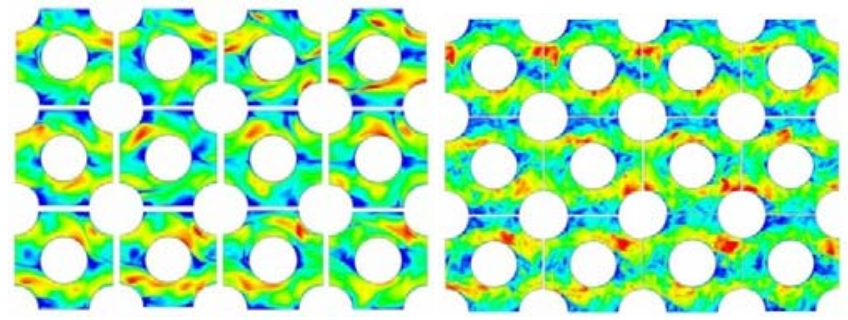

(c) LES/Smagorinsky(d) LES/WALE

FIGURE III. SUBSEQUENT VELOCITY MAGNITUDE CONTOURS FROM EACH METHOD.

Comparing the four types of computational results with the experimental data for the staggered tube bundles, it is shown that accuracy of the present MRT-LBM computation is comparable to theLES computations. Moreover, computing efficiencyin terms of elapsed time per one cell for the presentMRT-LBM is four to five orders of magnitude less than the other three computations.
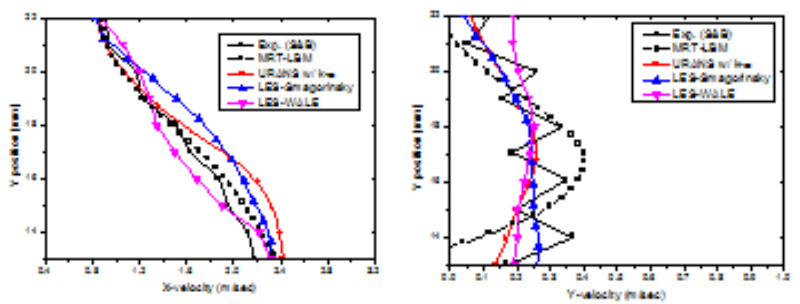

(a) Velocity profiles $x=0 \mathrm{~mm}$.
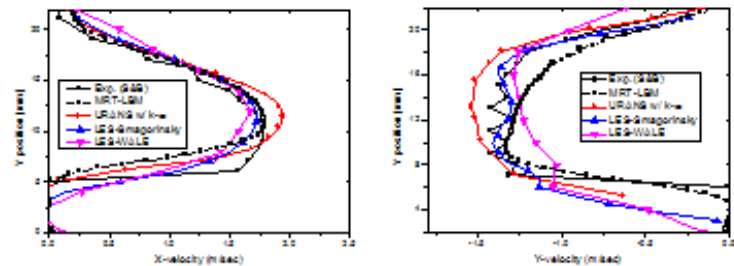

(b) Velocity profiles at $\mathrm{x}=11 \mathrm{~mm}$.
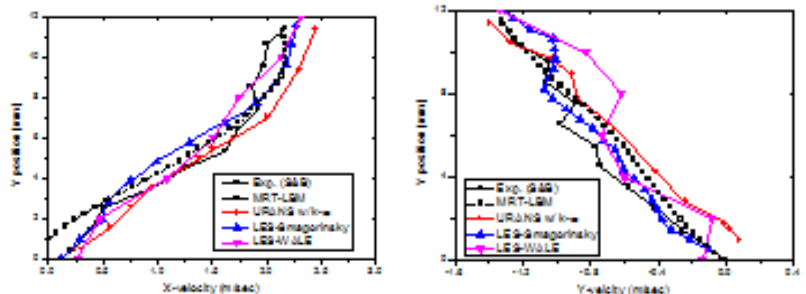

(c) Velocity profiles at $\mathrm{x}=16.5 \mathrm{~mm}$.
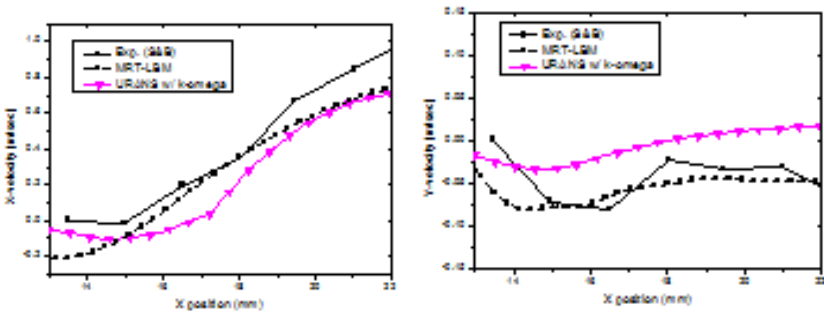

(d) Velocity profiles at $\mathrm{y}=0 \mathrm{~mm}$.
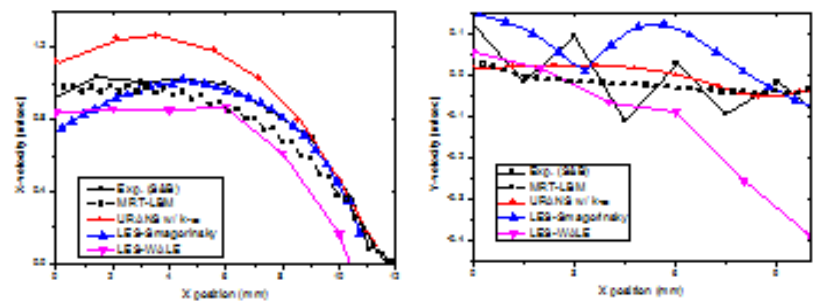

(e) Velocity profiles at $\mathrm{y}=22.5 \mathrm{~mm}$.

FIGURE IV.STREAM-WISE AND SPAN-WISE VELOCITY PROFILES

\section{CONCLUSIONS}

A two-dimensional computational fluid dynamic analysis code based on multi-relaxation time lattice Boltzmann method is developed and benchmarked against staggered tube bundles interested in the lower plenum of a very high temperature reactor. The MRT-LBM computations fora flow through the staggered tube bundles are also compared with general purpose CFD computationsusingthestandard k- $\omega$ turbulence and the large eddy simulation (LES) with turbulence closures of Smagrinsky-Lilly and WALE model. The agreement between the experimental and the present MRT-LBM computational results is reasonably acceptable for an engineering level of computation. Development of three dimensional in-house MRT-LBM codes based on D3Q15 and D3Q19 lattice models including thermal effects are underway.

\section{ACKNOWLEDGEMENTS}

This work was partially supported by Basic Atomic Energy Research Institute program (NRF-2010-0018759) and partially by the Nuclear Safety Research Program through the Korea Radiation Safety Foundation(KORSAFe), granted financial resource (Grant Code: 1305008-0113-HD120)from the Nuclear Safety and Security Commission(NSSC), Republic of Korea.

\section{REFERENCES}

[1] McIlroy Jr., H.M., McEligot, D.M.,Pink,R. J., Measurement of turbulent flow phenomena for the lower plenum ofa prismatic gas-cooled reactor. Nucl. Eng. and Des.,240, pp.416-428, 2010.

[2] Ridluan, A. and Tokuhiro,A., Benchmark Simulation of Turbulent Flow through a Staggered Tube Bundle to Support CFD as a Reactor Design Tool. Part II URANS CFD Simulation.J.ofNucl.Sci.and Tech.,45, pp.1305-1315, 2008.

[3] Moulinec, C., Pourqui'e, M. J. B. M., Boersma, B. J., Buchal, T., Nieuwstadt, F. T.M.,Direct numerical simulation on aCartesian mesh of the flow through a tube bundle.Int. J.Comput. Fluid Dyn,18,pp.1-14, 2004.

[4] Succi, S., The Lattice Boltzmann Equation for fluid dynamics and beyond, Clarendon Press: Oxford, 2001. 
[5] D’Humieres, D.,Ginzburg, I., Krafczyk, M., Lallemand, P.,Luo, L.-S., Multiple-relaxation-time lattice Boltzmann models in three dimensions. Phil. Trans. R. Soc. Lond. A, 360,pp.437-451, 2002.

[6] Smagorinsky, J. S., General circulation experiments with the primitive equations, part I: The basic experiment. Monthly Weather Review,91, pp.99-164, 1963.

[7] Simonin, O. and Barcouda, M., Measurements and prediction ofturbulent entering a staggered tube bundle.4th Int. Symp.Applicationsof Laser Anemometry to Fluid Mechanics, Lisbon,Portugal, paper 5.23, 1988.

[8] ANSYSInc.,ANSYS FLUENT Theory Guide,Ver. 14.0, Canonsburg, 2011.

[9] Yu, D., Mei, R., Luo, L.-S., Shyy, W., Viscous Flow Computations with the Method of Lattice Boltzmann Equation.Prog.Aerosp.Sci., 39, pp.329$367,2003$.

[10] Ho, C.-F., Chang, C., Lin, K.-H., Lin, C.A., Consistent Boundary Conditions for 2D and 3D LatticeBoltzmannSimulations.Tech Science Press CMES, 44, pp.137-155, 2009.

[11] Mei, R., Yu, D., Shyy, W., Force evaluation in the lattice Boltzmann method involving curved geometry. Phys. Rev., E, 65, 041203 (14pp), 2002.

[12] Mathworks, MATLAB Language Reference Manual, version 2013b, 2013.

[13] Langhans, W., Schmidli, J., Szintai,B., A Smagorinsky-Lilly turbulence closurefor COSMO-LES: Implementation and comparison to ARPS. COSMO-Newsletter, No.12, 2012.

[14] Ducros, F., Nicoud, F., Poinsot, T., Wall-Adapting Local EddyViscosity Models forSimulations in Complex Geometries. Proc. of 6th ICFD Conference on NumericalMethods for Fluid Dynamics, pp.293299, 1998. 